\title{
Communications
}

2018; 6(1): 20-24

$\mathrm{http}: / / \mathrm{www} \cdot \mathrm{sciencepublishinggroup.com} / \mathrm{j} / \mathrm{com}$

doi: $10.11648 /$ j.com. 20180601.14

ISSN: 2328-5966 (Print); ISSN: 2328-5923 (Online)

Communication

\section{Methods of Determining Reference Signals for One and Multichannel Signatural Analyzer of Microprocessor Systems}

\author{
Rustam Xusanovich Djuraev, Jo'shqin Boltabaevich Baltaev, Ulug'bek Baxodirovich Alimov \\ Department of Data Communication Networks and System, Telecommunication Faculty, Tashkent University of Information Technologies \\ Named After Muhammad Al-Khwarizmi, Tashkent, Uzbekistan
}

Email address:

jowkin@mail.ru (J. B. Baltaev)

\section{To cite this article:}

Rustam Xusanovich Djuraev, Jo'shqin Boltabaevich Baltaev, Ulug'bek Baxodirovich Alimov. Methods of Determining Reference Signals for One and Multichannel Signatural Analyzer of Microprocessor Systems. Communications. Vol. 6, No. 1, 2018, pp. 20-24.

doi: $10.11648 /$ j.com.20180601.14

Received: January 5, 2018; Accepted: February 7, 2018; Published: March 14, 2018

\begin{abstract}
The article deals with methods for determining signatures for signature analysis in the diagnosis of microprocessor systems.It is shown that the main document of signature analysis is the dictionary of reference signatures. The analysis of methods for the determination of reference signatures used in the diagnosis of microprocessor systems (MPS) is carried out.A program for simulating one and multi-channel signature method for automating the definition of reference signatures.
\end{abstract}

Keywords: Signature Analysis (SA), Microprocessor Devices, Microprocessor Systems (MPS),

Single-Channel Signature Analysis (SSA), Multichannel Signature Analysis (MSA), Signatura

\section{Introduction}

Transition to the wide use of IPC in modern digital systems has created, together with indisputable advantages, a number of serious problems in their maintenance, related primarily to the monitoring and diagnostic processes. In general, up to $70-80 \%$ of the recovery time of failed digital systems is the time of technical diagnostics, consisting of the time of search and localization of the failed elements. [1, 2]

The classical strategy of testing digital systems, based on the formation of test sequences, requires significant time and large amounts of test information and standard output reactions, which implies the availability of complex equipment. The most effective solution, allowing to significantly reduce the amount of stored information about the reference output reactions, is a single and multi-channel signature diagnostic method in digital circuits. [3, 4]

An important parameter of signature analysis is a set (dictionary) of reference signatures, which is predetermined for a functioning digital device. The need to create methods for calculating signatures is associated with the need to automate the production of dictionaries (tables) of reference signatures, since their creation by means of signature measurement is rather time-consuming. Therefore, the main document of signature analysis is the dictionary of reference signatures, which defines an algorithm for troubleshooting.

Thus, the need to create methods for calculating signatures is connected first with the assessment of the reliability of the SA, and secondly, with the need to automate the production of dictionaries (tables) of reference signatures, which are the main document that determines the algorithm for troubleshooting.

At the present time, various theoretical methods are known that allow one to calculate reference signatures $[5,6]$.

\section{Method for Calculating Reference Signatures Based on Nested Polynomials}

Let's consider the scheme of the generator of pseudo-random sequences on the n-bit shift register, with feedbacks taken from $n$, $\mathrm{m}, 1, \mathrm{k}$ bits, which are put in the adder modulo 2. [7-8]. 


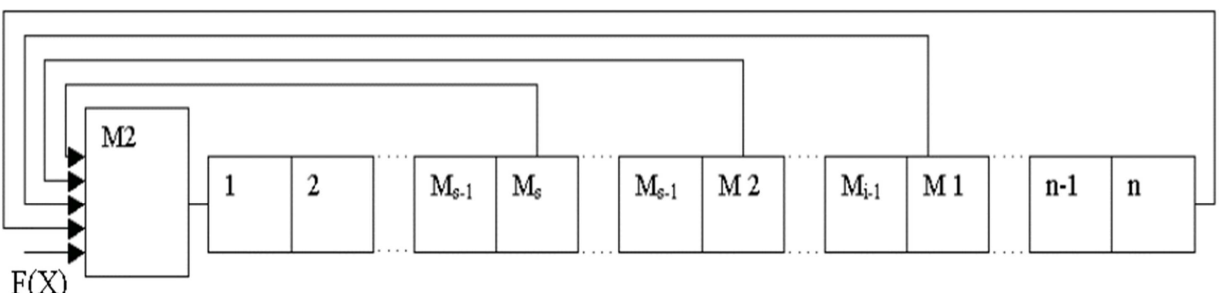

Figure 1. Scheme of the pseudo-random sequence generator (CST) based on a shift register of $n$-bit length.

Then

$$
F(X)=g(X) \cdot P_{n}(X)+r(X)
$$

where $\mathrm{F}(\mathrm{X})$ is the input polynomial;

$\mathrm{g}(\mathrm{X})$ - quotient;

$\mathrm{P}_{\mathrm{n}}(\mathrm{X})$ - inverse of the feedback polynomial for the n-bit register;

$\mathrm{r}(\mathrm{X})$ - remnant.

In [4], the shift register is considered as a set of nested shift registers of length $\mathrm{n}, \mathrm{m}, \mathrm{l}, \mathrm{k}$ digits. It is obvious that each register performs its own division (transformation) of the input polynomial. If the degree of the input polynomial is greater than the degree of the shift register, then the effect of nested registers is taken into account in the feedback polynomial of the shift register of a larger length. If the degree of the input polynomial does not exceed the degree of the register, then the input polynomial divides into the inverse of the inverse polynomial, the shift register of smaller length. Since the degree of $r(X)$ is less than the degree of $P(X)$, to obtain the real remainder, it is necessary to divide $\mathrm{r}(\mathrm{X})$ by the inverse of the polynomials of feedbacks of registers of smaller length. [9-10].

We denote the inverse polynomial in terms of $\mathrm{P}_{\mathrm{n}}^{\prime}(\mathrm{X}), \mathrm{P}_{\mathrm{T}}^{\prime}(\mathrm{X}), \mathrm{P}_{1}^{\prime}(\mathrm{X}), \mathrm{P}_{\mathrm{k}}^{\prime}(\mathrm{X})$. We divide the input polynomial $\mathrm{F}(\mathrm{X})$ by the polynomial:

$$
\frac{F(X)}{P_{n}^{\prime}(X)}=g_{n}(X)+\frac{r_{n}(X)}{P_{n}^{\prime}(X)}
$$

Then we consider consecutively the remainders $r_{i}(X)$, $(\mathrm{i}=\mathrm{n}, \mathrm{m}, \mathrm{l})$ as input polynomials for shift registers of smaller length, dividing them by polynomial:

$$
\begin{aligned}
& \frac{r_{n}(X)}{P_{m}^{\prime}(X)}=g_{m}(X)+\frac{r_{m}(X)}{P_{m}^{\prime}(X)} \\
& \frac{r_{m}(X)}{P_{1}^{\prime}(X)}=g_{1}(X)+\frac{r_{1}(X)}{P_{1}^{\prime}(X)} \\
& \frac{r_{1}(X)}{P_{k}^{\prime}(X)}=g_{k}(X)+\frac{r_{k}(X)}{P_{k}^{\prime}(X)}
\end{aligned}
$$

Analysis of formulas $(1-4)$ shows that $g_{n}(X)$ is an output polynomial of the degree register $n, g_{m}(X)-$ The output polynomial of the register of degree $\mathrm{m}$, which fills the digits from $\mathrm{m}+1$ before $\mathrm{n}$ shift register, $\mathrm{g}_{1}-$ output polynomial of the register of degree 1, which fills the bits from $1+1$ to $\mathrm{m}$, etc.;
$r_{k}(X)$ - the remainder remaining in the shift register, which has only one feedback and fills the bits from 1 to $\mathrm{k}$. Thus, the signature can be written in the following form:

$$
r_{c}(X)=g_{m}(X) X^{m+1}+g_{1}(X) X^{1+1}+g_{k}(X) X^{k+1}+r_{x}(X) X
$$

By this formula, it is possible to calculate the signatures for the shift register with a numbering from 1 to $n$. If the numbering of the digits starts from zero, then formula (5) takes the form:

$$
r_{c}(X)=g_{m}(X) X^{m}+g_{1}(X) X^{1}+g_{k}(X) X^{k}+r_{x}(X)
$$

So for the CEP, developed by HP, the formula (6) has the form:

$$
r_{c}(H P)=g_{12}(X) X^{12}+g_{9}(X) X^{9}+g_{7}(X) X^{7}+r_{7}(X)
$$

Let's consider an example of calculation of a signature by the specified method for an input sequence. 11111100000111111111 dividing the input sequence $\mathrm{F}(\mathrm{X})$ on the inverse polynomial $P^{\prime}(X)$ we obtain the: $g(X)=x^{3}+x^{2}+x$ +1 and the remainder $r_{16}(X)=x^{15}+x^{14}+x^{12}+x^{11}+x^{7}$. Then we divider ${ }_{16}(\mathrm{X})$ on the inverse of the feedback polynomial of the 12-bit register $\mathrm{P}(\mathrm{X})=\mathrm{x}^{12}+\mathrm{x}^{5}+\mathrm{x}^{3}+1$. As a result, we obtain the $\mathrm{g}_{12}(\mathrm{X})=\mathrm{x}^{3}+\mathrm{x}^{2}+1$ and the remainderr ${ }_{12}(\mathrm{X})=\mathrm{x}^{11}+$ $x^{9}+x^{6}+x^{2}+1$. Dividing $r 12(X)$ on the inverse of the feedback polynomial of the 9-bit register. As a result, we obtain the

$$
\mathrm{g}_{9}(X)=\mathrm{x}_{2} \text { and the remainder } \mathrm{r}_{9}(X)=\mathrm{x}^{8}+\mathrm{x}^{6}+\mathrm{x}^{4}+1 \text {. }
$$

Dividing $r_{9}(X)$ on the inverse of the feedback polynomial of the 7-bit register.

As a result, we obtain the $g_{7}(X)=X$ and the remainder $r_{7}(X)=x^{6}+x^{4}+x+1$.

Substituting these values into the expression for $r_{c}(X)$, we obtain

$r_{c}(X)=\left(x^{3}+x^{2}+1\right) x^{12}+x^{2} x^{9}+x^{8}+x^{7}+x^{6}+x^{4}+x+1=x^{15}+$
$x^{14}+x^{12}+x^{11}+x^{8}+x^{6}+x^{4}+x+1$

We transform the obtained polynomial into the binary form $r_{c}(H P)=1101100101010011$, to which the signature H953

\subsection{Method for Calculating Reference Signatures Based on Analysis of the SSA Operation}

As you know, the essence of SSA is that the data sequences from the node, the correctly functioning circuit in the test 
mode are put in accordance with a certain signature. In the subsequent verification of this scheme, the operator uses the analyzer to measure the signatures at various points in the circuit of the digital device and compare them to the standard signatures recorded in the documentation. The principle of the $\mathrm{SA}$ is based on the method of signature analysis, that is, the compression of long sequences into four-digit hexadecimal signatures. Physically, this method is implemented on a linear shift register with feedbacks, whose signals are summed modulo two with the input sequence.

The polynomial is an irreducible polynomial

$$
P(X)=x^{16}+x^{12}+x^{9}+x^{7}+1
$$

The signature is formed using a shift register with a logical feedback $P(X)=x^{16}+x^{12}+x^{9}+x^{7}+1$, at the input of which there is an adder modulo two.

Suppose that during the connection of the probe SA to a control point, a 20-bit sequence of ones and zeros appeared in it, having the form: 11111100000111111111.

This input sequence is summed modulo 2 with the contents of the shift register cells 7, 9, 12 and 16.After 20 clock cycles of operation of the circuit, the 16-bit combination 1101100101010011 will be in the register, which, as a result of division into four four-digit combinations, corresponds to the alphanumeric signature H953.

\subsection{Method for Determining Signatures Based on the Modeling of a Single-Channel Signature Analyzer}

The program for modeling the signature method for diagnosing digital devices SIGNATURA is designed to visually display the process of forming a digital signature when diagnosing the device [8].The program simulates the process of passing a digital sequence through a logic circuit consisting of a shift register and an adder modulo 2. After the simulation process is over, the program outputs the result - a digital signature in the form of a code for displaying on a seven-segment indicator. The program works in the operating systems Microsoft Windows NT / 2000 / XP / 2003/7/10.

The program does not require installation and is launched by running the signatura.exe application. When you start, the program window appears. [11-12].

The program window contains the image of the simulated circuit in the upper part, the control elements at the bottom. [13].

The sequence of work with the program is as follows:

- set the digit of the shift register;

- set the bits of the polynomial;

- enter the input sequence;

- if necessary, set the interval for acceleration / deceleration of the process;

- Click the "Start" button.

When the "Start" button is pressed, the input sequence starts to be fed to the input of the adder modulo 2.The bits of the input sequence pass through the logic circuit and are written to the shift register.

After passing through all the bits of the input sequence through the logic, a digital signature appears on the screen, which is a code for the seven-segment indicator (Figure 2).

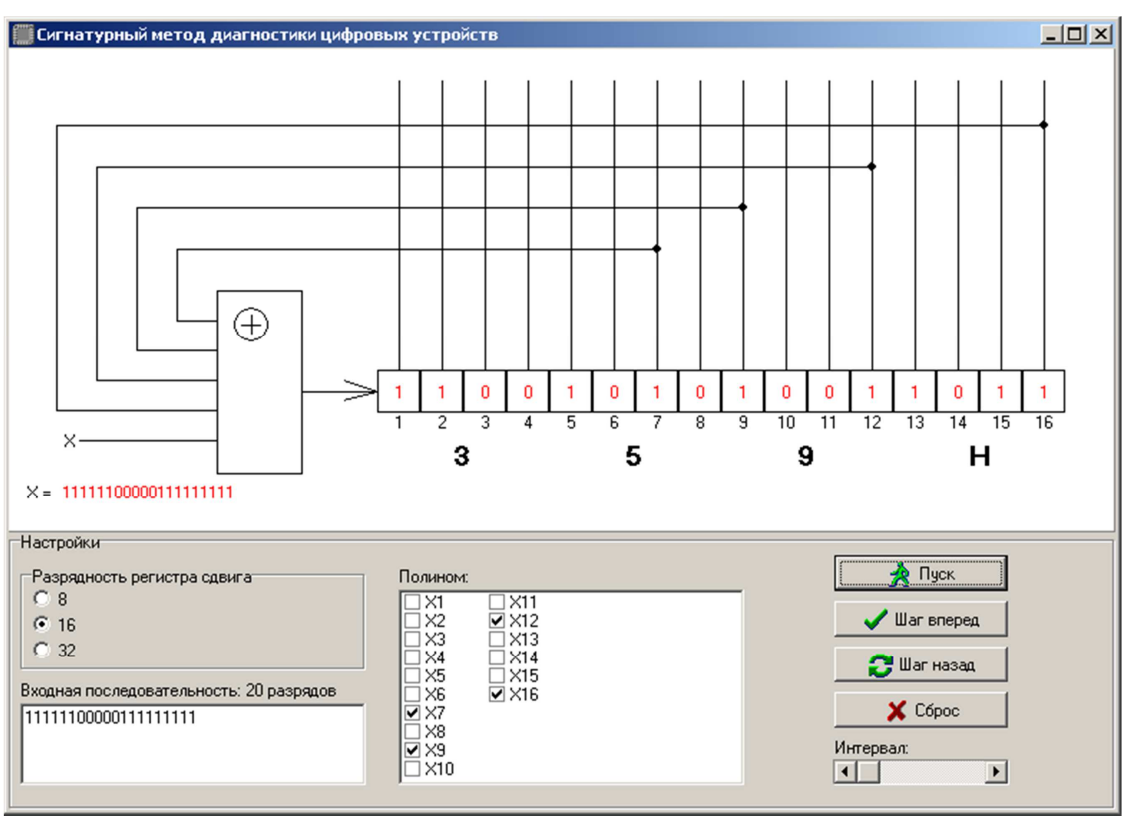

Figure 2. Digital signature after the end of the process.

\section{Result}

Each symbol of the signature is displayed under the corresponding four bits of the shift register. The six-digit register corresponds to a four-character signature, eight-digit - two-character, thirty-two-digit - eight-character. Method for determining signatures based on the MSA simulation. An analysis of the n-output digital circuit by a single-channel signature analyzer (SA) leads to an increase in $n$ times of the time required for circuit analysis or equipment required to implement $\mathrm{n}$ signature analyzers. Multichannel signature 
analyzers allow significantly speeding up the procedure for monitoring digital circuits, which practically increases by $n$ times, where $\mathrm{n}$ is the number of inputs of the analyzer used.

The program for modeling the work of the MSA is written in PHP and with the help of the program Devel Studio the code of the program was placed in a separate file, which does not require a virtual server to work.

This program simulates the operation of a multichannel SA for 4.8.16 channels, calculates convolutions. In order to calculate the convolution, one must:

1) introduce the sequence vectors.

2) then specify their length (the "Length" field)

3) after you enter the polynomial which is responsible for the feedback in the shift register (the "polynomial" field).

4) then click start.

The program window for modeling the work of the MSA.

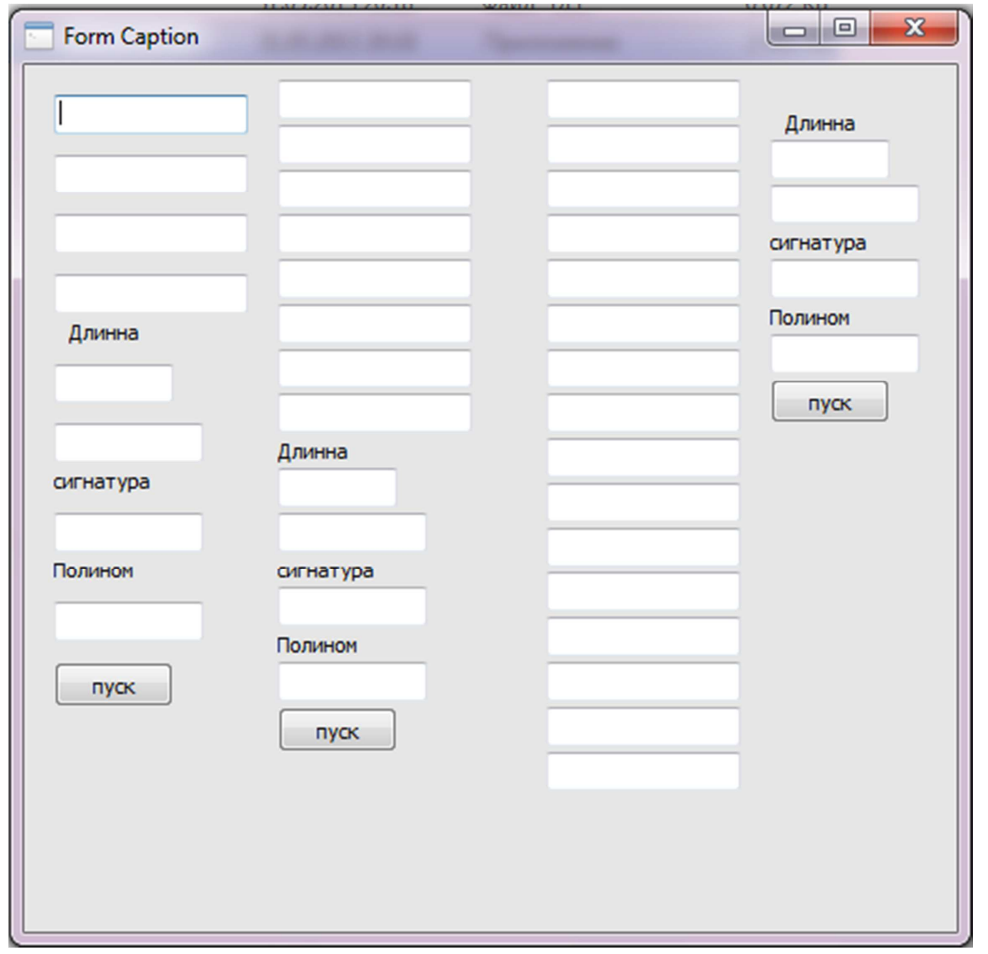

Figure 3. Next, the result of the program, i.e.it allows to calculate the reference signatures for the 4.8.16 channel MSA.

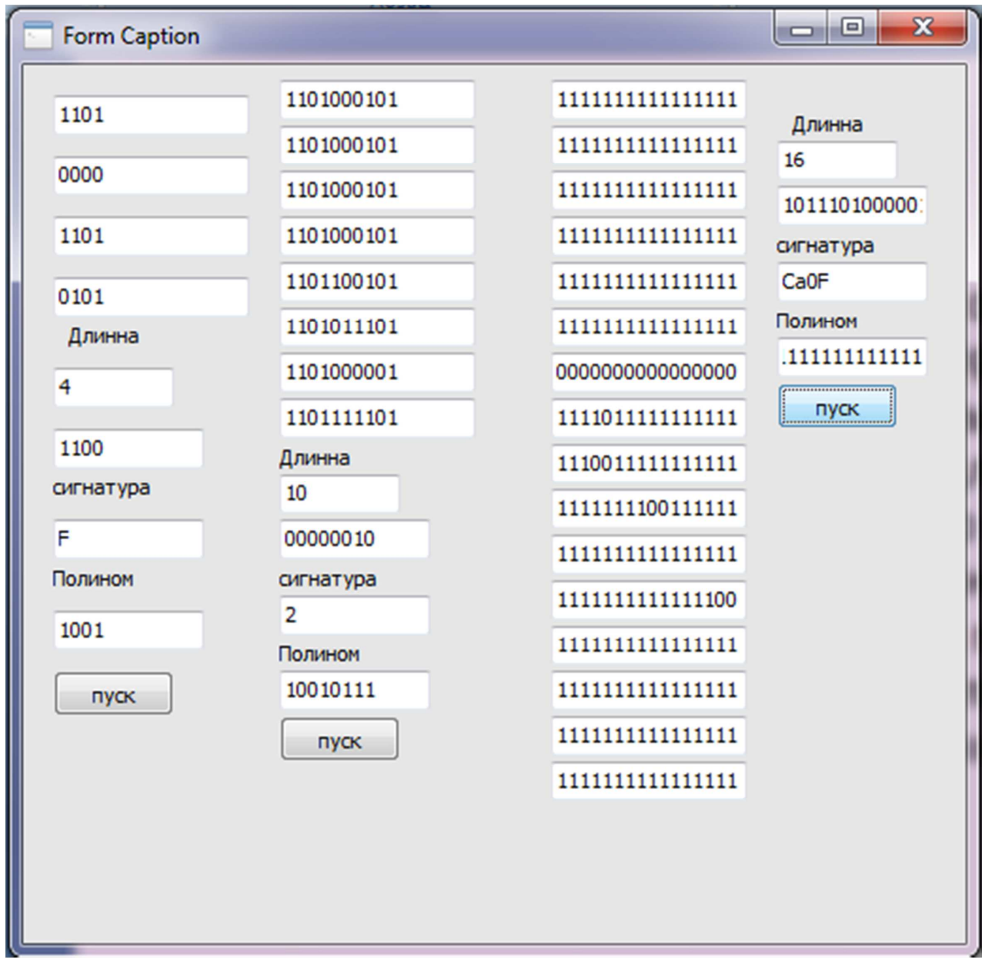

Figure 4. MSA result. 


\section{Conclusion}

Based on the conducted studies, the following conclusions can be drawn:

- an analysis of methods for determining reference signatures for SA in the diagnosis of MPS;

- for the purpose of automating the definition of reference signatures, algorithms and simulation programs for single and multi-channel signature method for diagnosing digital devices have been developed;

- developed software for modeling SSA works in the operating systems Microsoft Windows NT / 2000 / XP / 2003/7/10.

- The program for modeling the work of the MSA is written in the language of PHP and using the program Devel Studio.

\section{References}

[1] Aripov M. N., Djuraev R. X., Technical diagnostics of digital systems. Tutorial Tashkent2006, str.2.

[2] Djuraev R. X., Djabbarov Sh. Yu., Baltaev J. B. “Analysis of the model of malfunction and diagnostics of multi-output digital devices by a multichannel signature analyzer" ToshDTU Xabarlari №3, 2013, str. 55-60.

[3] Djuraev R. X., Djabbarov Sh. Yu., Yangaliev F. Sh., Program for modeling the signature method for diagnosing digital devices. //№ DGU 01244,26.03.2007.

[4] Djabbarov Sh. Yu., Djuraev R. X., Baltaev J. B. "Simulation of multi-channel signature method for diagnosis in multimedia terminal devices" CONFERENCE PROCEEDINGS MITA
2015. June 30-July 2, 2015, Tashkent, Uzbekistan. 139-142 rr.

[5] Djuraev R. X., Djabbarov Sh. Yu., Baltaev J. B. The program of modeling of four-channel, eight-channel and sixteen-channel signature method of diagnostics of digital devices. //№ DGU 04122, № DGU 04123, № DGU 0412408.11.2016.

[6] Bestugin A. R., Bogdanova G. F., StogovG. B. Monitoring and Diagnostics of Telecommunication Networks - SPb: Politexnika, 2003. 174 s.: il.

[7] Bayda N. P., Kuzmin I. V., Shpilevoy V.T. Microprocessor-based element diagnosis systems REA. - M.: Radio i svyaz, 1987.-256s.

[8] Djabbarov Sh.Yu.,ToshDTU xabarlari "Principles of diagnostics of digital devices by means of signature analysis" №2. 2006.

[9] GnedovG.M. Control of data transmission equipment. -M .: Radio and communication, 1983.-152s.

[10] Gulyaev V.A., Kudryashov V.I. Automation of the setup and diagnosis of micro-UAK.-M.:Energoatomizdat, 1992.-256s.

[11] Davidov P.S. Technical diagnostics of radio electronic devices of systems. -M .: Radio and communication, 1988.-256s.

[12] Djeykoks Dj. Guide to troubleshooting electronic equipment. Translation from English. - M.: Mir, 1989.-176s.

[13] Kazmina S. K. Compact testing // Automation and telemechanics, 1982, N3, s.173-187.

[14] Lvovich G. A., Samoshenko A. V., Xavkin V. E. Diagnosis of microprocessor systems. -Reviews on electronic engineering. Ser.3.Microelectronics, vip.7. -M.:SNII "Elektronika", 1987.-84s. 